\title{
Loop Shifting and Non-Conservative qLPV Design
}

\author{
József Bokor $^{1}$ and Zoltán Szabó ${ }^{2}$ \\ ${ }^{1}$ MTA-BME Control Engineering Research Group, Computer and Automation \\ Research Institute, Hungarian Academy of Sciences, Hungary; e-mail: \\ bokor@sztaki.hu \\ ${ }^{2}$ Computer and Automation Research Institute, Hungarian Academy of Sciences, \\ Hungary; e-mail: szaboz@ sztaki.hu
}

\begin{abstract}
As an extension of the robust $H_{\infty}$ theory, the time domain design based on linear matrix inequalities (LMI) is a conceptually simple and efficient framework to obtain qLPV controllers. However, the constructed scheduling variables are not always suitable for an efficient implementation. This paper investigates the possibility of constructing the scheduling block of a qLPV controller explicitly, i.e., in the form of a linear fractional transformation (LFT). It is shown here that if both the primary and dual multiplier LMI equations lead to maximal indefinite subspaces and a coupling condition holds, the problem can be solved and a constructive algorithm results to build the desired scheduling variables.
\end{abstract}

Keywords: Linear Fractional Transform; robust control; qLPV design

\section{Introduction and Motivation}

In modern control design a quasi-linear parameter varying (qLPV) description is frequently used [1]. The approach is based on the possibility of rewriting the plant in a form in which nonlinear terms can be hidden by using suitably defined scheduling variables by maintaining the linear structure of the model. An advantage is that in the entire operational interval nonlinear systems can be defined and a well-developed linear system theory, to analyze and design nonlinear control systems, can be used.

The models are augmented with performance specifications and uncertainties. Weighting functions are applied to the performance signals to meet performance specifications and guarantee a good tradeoff between performance results. The uncertainties are modeled by both un-modeled dynamics and parametric uncertainties. As a result of this construction a linear fractional transformation (LFT) interconnection structure, which is the basis of control design, is achieved. In this particular structure, also known as a $M-\Delta$ configuration, where the 
parameter-varying terms (scheduling variables) are located in the diagonal of the $\Delta$ operator and the time invariant part is described by the linear operator $M$.

In the design of robust linear parameter varying controllers, the LMI-based linear time invariant (LTI) robust control design framework has a central role, [2], [7], [8], [9], [10], [16], [17]. As opposed to the gain scheduling technique these approaches provide a design algorithm that starts from an analysis equation that guarantees a certain (quadratic) performance level and the designed controller is supposed to fulfill the robust stability and performance requirements. Therefore, qLPV models with linear matrix inequalities (LMI), as the main design tool, seem to be the most efficient approach to achieve robust and non-conservative results.

While the main steps of the quadratic robust design are well-known, there are still a lot of questions concerning the practical construction. Many of the methods, in order to keep the design linear, restrict the multipliers of the scheduling variables to a predefined set, which makes these methods conservative in general.

In this paper, the control design problem is set in the framework presented in [9], [10], [11], that strongly exploits the available LMI techniques. As opposed to the gain scheduling technique these approaches provide a design algorithm that starts from an analysis equation that guarantees a certain (quadratic) performance level and the designed controller is supposed to fulfill the robust stability and performance requirements. The method has a certain level of conservatism due to the use of constant multipliers; however it can be the starting point of more elaborate designs [12].

Our interest in this method is motivated by both theoretical and practical issues. On a theoretical level this method is a direct extension of the $\mathcal{H}_{\infty}$ design theory to an LPV context. The LTI part of the controller and the scheduling block of the controller are obtained through a similar extension process as the controller itself in the original $\mathcal{H}_{\infty}$ setting. However, the extension related to the scheduling block is related to indefinite matrices that makes the process more challenging.

On the practical side the problem concerns the scheduling variables of the controller. In the general setting, i.e., when there are no restrictions imposed on certain blocks of the multipliers, the construction of the scheduling block involves an on-line computation of a spectral subspace of a time-varying matrix, which is a computationally demanding task for the implementation. It is preferred to obtain this block in an explicit form, e.g., as an LFT of the original scheduling variables. Thus, a post-processing method, like the tensor product transformation method [13] is needed.

The aim of the paper is to investigate the possibility of constructing the scheduling block of a LPV controller explicitly. It is shown that if both the primary and dual multiplier LMI equations leads to maximal indefinite subspaces and admits a common "loop-shifting", then the problem can be solved. Once this common "loop-shifting" exists, the proposed approach is constructive and an algorithm is also provided to build the desired block of scheduling variables. 
For the sake of completeness, Section 2 provides a short overview of the classical LPV controller construction presented in [9] and [10]. The analysis and the development of the proposed method are contained in Section 3. The main idea exploited in the paper resembles the idea of the loop-shift in the classical control design; this motivates the title of the section. The analysis also contains clarifications and additions concerning the original problem setting treated in [9] and [10] that might help the deeper understanding of the different conditions involved in these algorithms.

\section{Problem Setup}

In this section we summarize the basic setup according to [10] and to ease the understanding we barrow the notations from that paper. Accordingly, the open loop generalized plant is defined as:

$$
\begin{aligned}
& \left(\begin{array}{l}
\dot{x}(t) \\
z_{u}(t) \\
z_{p}(t) \\
y(t)
\end{array}\right)=\left(\begin{array}{llll}
A & B_{u} & B_{p} & B \\
C_{u} & D_{u u} & D_{u p} & E_{u} \\
C_{p} & D_{p u} & D_{p p} & E_{p} \\
C & F_{u} & F_{p} & 0
\end{array}\right)\left(\begin{array}{l}
x(t) \\
w_{u}(t) \\
w_{p}(t) \\
u(t)
\end{array}\right) \\
& \left(\begin{array}{l}
w_{u}(t) \\
z_{u}(t)
\end{array}\right) \in \mathcal{S}(\Delta(t)) \subset \mathbb{R}^{m_{u}+k_{u}}
\end{aligned}
$$

while the output--feedback LPV controller for (1) is described as

$$
\begin{aligned}
& \left(\begin{array}{l}
\dot{x}_{c}(t) \\
u(t) \\
z_{c}(t)
\end{array}\right)=\left(\begin{array}{lll}
A_{c} & B_{c 1} & B_{c 2} \\
C_{c 1} & D_{c 11} & D_{c 12} \\
C_{c 2} & D_{c 21} & D_{c 22}
\end{array}\right)\left(\begin{array}{l}
x_{c}(t) \\
y(t) \\
w_{c}(t)
\end{array}\right) \\
& \left(\begin{array}{l}
w_{c}(t) \\
z_{c}(t)
\end{array}\right) \in \mathcal{S}_{c}(\Delta(t)) \subset \mathbb{R}^{m_{c}+k_{c}},
\end{aligned}
$$

where the on-line measured parameter satisfy $\Delta(t) \in \nabla$. As opposed to the original setting it is assumed that $\mathcal{S}(\Delta)$ and $\mathcal{S}_{c}(\Delta)$ admit the explicit description $\operatorname{Im}\left(\begin{array}{c}\Delta \\ I_{k_{u}}\end{array}\right)$ and $\operatorname{Im}\left(\begin{array}{c}\Delta_{c} \\ I_{k_{c}}\end{array}\right)$, respectively. Under the standing hypothesis of well-posedness and of continuity these assumptions do not restrict the generality.

The controller should fulfill the quadratic $\mathcal{H}_{\infty}$ performance, where the performance index $\gamma$ is an indicator on the quality of the controller. 
Theorem 1 (LPV analysis) There exist a controller (2) such that closed-loop system is well-posed and stable if and only if there exist $X, Y$, multipliers $P=\left(\begin{array}{ll}Q & S \\ S^{T} & R\end{array}\right)$ and $\tilde{P}=\left(\begin{array}{ll}\tilde{Q} & \tilde{S} \\ \tilde{S}^{T} & \tilde{R}\end{array}\right)$ with $P>0$ on $\mathcal{S}(\Delta)$ and $\tilde{P}<0$ on $\mathcal{S}(\Delta)^{\perp}$ for all $\Delta \in \nabla$ that satisfy the matrix inequalities

$$
\begin{aligned}
& \left(\begin{array}{ll}
X & I \\
I & Y
\end{array}\right) \geq 0 \\
& \Psi^{T}\left(\begin{array}{l}
* \\
* \\
*
\end{array}\right)^{T}\left(\begin{array}{llllll}
0 & X & 0 & 0 & 0 & 0 \\
X & 0 & 0 & 0 & 0 & 0 \\
0 & 0 & Q & S & 0 & 0 \\
0 & 0 & S^{T} & R & 0 & 0 \\
0 & 0 & 0 & 0 & Q_{p} & S_{p} \\
0 & 0 & 0 & 0 & S_{p}^{T} & R_{p}
\end{array}\right)\left(\begin{array}{lll}
I & 0 & 0 \\
A & B_{u} & B_{p} \\
0 & I & 0 \\
C_{u} & D_{u u} & D_{u p} \\
0 & 0 & I \\
C_{p} & D_{p u} & D_{p p}
\end{array}\right) \Psi<0, \\
& \Phi^{T}\left(\begin{array}{l}
* \\
* \\
*
\end{array}\right)\left(\begin{array}{llllll}
0 & Y & 0 & 0 & 0 & 0 \\
Y & 0 & 0 & 0 & 0 & 0 \\
0 & 0 & \tilde{Q} & \tilde{S} & 0 & 0 \\
0 & 0 & \tilde{S}^{T} & \tilde{R} & 0 & 0 \\
0 & 0 & 0 & 0 & \tilde{Q}_{p} & \tilde{S}_{p} \\
0 & 0 & 0 & 0 & \tilde{S}_{p}^{T} & \tilde{R}_{p}
\end{array}\right)\left(\begin{array}{lll}
-A^{T} & -C_{u}^{T} & -C_{p}^{T} \\
I & 0 & 0 \\
-B_{u}^{T} & -D_{u u}^{T} & -D_{p u}^{T} \\
0 & I & 0 \\
-B_{p}^{T} & -D_{u p}^{T} & -D_{p p}^{T} \\
0 & 0 & I
\end{array}\right) \Phi>0
\end{aligned}
$$

where $\Phi=\operatorname{Ker}\left(\begin{array}{lll}B^{T} & E_{u}^{T} & E_{p}^{T}\end{array}\right)$ and $\Psi=\operatorname{Ker}\left(\begin{array}{lll}C & F_{u} & F_{p}\end{array}\right)$.

Usually conditions $R>0$ and $\tilde{Q}<0$ are also imposed. These conditions follow if we require that $0 \mathrm{ha}$. In the original construction $\Delta_{c}(0)=0$ is also supposed in order to get to the necessary inertia conditions, however, this requirement is not a natural one.

Controller synthesis starts with the solution of the analysis LMIs of Theorem 1 which usually involves a relaxation step, resulting in the matrices $X, Y$ and $P, \tilde{P}$.

Then the Lyapunov matrix $X_{e}$ of the closed-loop system can be obtained as

$$
X_{e}=\left(\begin{array}{lc}
X & Z \\
Z^{*} & {\left[Z^{*}\left(X-Y^{-1}\right) Z\right]^{-1}}
\end{array}\right),
$$

where $\operatorname{Im} Z=\operatorname{Im}\left(X-Y^{-1}\right)$. The multiplier $\mathcal{P}$, corresponding to the scheduling variables, can be obtained from $P$ and $\widetilde{P}$ as follows:

$P_{e}:=\left(\begin{array}{cc}P & U T \\ (U T)^{T} & T^{T}\left[U^{T}\left(P-\tilde{P}^{-1}\right) U\right]^{-1} T\end{array}\right)$.

$U$ is an orthogonal matrix such that $\operatorname{Im} U=\operatorname{Im}\left(P-\widetilde{P}^{-1}\right)$ and $T$ is a suitable nonsingular matrix, for additional details see [10]. 
The LTI part of the LPV controller can be obtained by solving the quadratic matrix inequality:

$$
\left(\begin{array}{c}
I_{m} \\
\mathcal{C}+\mathcal{A} \times \mathcal{B}
\end{array}\right)^{T} \mathcal{M}\left(\begin{array}{c}
I_{m} \\
\mathcal{C}+\mathcal{A} \times \mathcal{B}
\end{array}\right)<0,
$$

where $\mathcal{C} \in \mathbb{R}^{n \times m}, \mathcal{A} \in \mathbb{R}^{n \times k}, \mathcal{B} \in \mathbb{R}^{l \times m}$ are matrices that depend on the system matrices of the generalized plant $M$. The unknown is $\mathcal{X}=\left(\begin{array}{ll}A_{c} & B_{c} \\ C_{c} & D_{c}\end{array}\right) \in \mathbb{R}^{k \times l}$ and the multiplier $\mathcal{M}$ is assembled from $X_{e}, P_{e}$ and the performance multiplier $P_{p}=\left(\begin{array}{cc}-\gamma I & 0 \\ 0 & I\end{array}\right)$. A numerically reliable algorithm and a parametrization of the solutions of this inequality was given by the authors in [14].

\subsection{Scheduling Variables}

The most delicate point of the algorithm is the construction of the scheduling variables. If we have for the blocks $Q<0$ and $\tilde{R}>0$ the multipliers $P$ and $\tilde{P}$ of Theorem 1, then there is an explicit construction [9].

The key point is that the extended multiplier should inherit this property. This indeed is possible by setting

$P_{e}:=\left(\begin{array}{cc}P & T \\ T^{T} & T^{T} N T\end{array}\right)$,

with $N=\left(P-\tilde{P}^{-1}\right)^{-1}$ and $T=\left(T_{1} T_{2}\right)$ having the blocks $T_{1}=T W, T_{2}=T \widetilde{W}$, where $W=\left(\begin{array}{l}I \\ 0\end{array}\right), \widetilde{W}=\left(\begin{array}{l}0 \\ I\end{array}\right)$.

These blocks are chosen to fulfill the conditions

$T_{1}^{T}\left(N-W Q^{-1} W^{T}\right) T_{1}<0$,

$T_{2}^{T}\left(N-\widetilde{W} R^{-1} \widetilde{W}^{T}\right) T_{2}>0$.

By permuting the blocks of $P_{e}$ one has the partitioning $\left(\begin{array}{ll}Q_{e} & S_{e} \\ S_{e}^{T} & R_{e}\end{array}\right)$ with $Q_{e}<0$ and $R_{e}>0$. The scheduling block $\Delta_{c}$ of the controller can be obtained from the condition

$\left(\begin{array}{llll}U_{11} & U_{12} & \left(W_{11}+\Delta\right)^{T} & W_{21}^{T} \\ U_{21} & U_{22} & W_{12}^{T} & \left(W_{22}+\Delta_{c}\right)^{T} \\ W_{11}+\Delta & W_{12} & V_{11} & V_{12} \\ W_{21} & W_{22}+\Delta_{c} & V_{21} & V_{22}\end{array}\right)>0$

where

$U=R_{e}-S_{e}^{T} Q_{e}^{-1} S_{e}, V=-Q_{e}^{-1}, W=Q_{e}^{-1} S_{e}$,

as 
$\Delta_{c}=-W_{22}+\left(\begin{array}{ll}W_{21} & V_{21}\end{array}\right)\left(\begin{array}{ll}U_{11} & W_{11}^{T}+\Delta^{T} \\ W_{11}+\Delta & V_{11}\end{array}\right)^{-1}\left(\begin{array}{l}U_{12} \\ W_{12}\end{array}\right)$,

for additional details see [9].

We remark here, that the condition $\Delta_{c}(0)$ is not supposed and does not hold in general. Thus condition $R_{e}>0$ is a simple choice that facilitates the design. Also, note the size of the scheduling block which is twice the original. This is not surprising if one recalls that in an LPV setting, both the state feedback and the observer gain, is parameter varying. Putting together the two blocks one can arrive to the size from (12). For the dynamics we do not meet this effect, since the state feedback has no dynamics.

Since $R>0$ and $\tilde{Q}<0$ is always imposed for the design the relevant conditions are $Q<0$ and $\tilde{R}>0$ to use this scheme. For a convex relaxation scheme, i.e., when the relevant LMIs are imposed at the vertices of the polytope defined by the scheduling variables, these conditions should hold. For the role of the choice of the convex-hull and other related relaxations schemes that can also be applied [13]. The choice of a proper relaxation scheme is the corner-stone of a successful control design, thus to decrease conservatism one should use multipliers with an indefinite $Q$.

The procedure to construct the scheduling variables in the general case is described in [10], and unfortunately, is quite involved. Since it involves projections on an eigenspace of a parameter varying matrix, the scheduling variable is not in explicit form, that is actually a linear fractional transform (LFT), as in Eq. (12). Therefore it is a quest for extend the applicability of this simple design for a more general setting. In what follows a partial answer to this problem will be given.

\section{Loop Shifting}

The starting LMI for obtaining the scheduling variables is

$\left(\begin{array}{ll}\Delta & 0 \\ 0 & \Delta_{c} \\ I & 0 \\ 0 & I\end{array}\right)^{T}\left(\begin{array}{ll}Q_{e} & S_{e} \\ S_{e}^{T} & R_{e}\end{array}\right)\left(\begin{array}{cc}\Delta & 0 \\ 0 & \Delta_{c} \\ I & 0 \\ 0 & I\end{array}\right)>0$,

i.e., $\left(\begin{array}{c}\bar{\Delta} \\ I\end{array}\right)^{T} P_{e}\left(\begin{array}{c}\bar{\Delta} \\ I\end{array}\right)>0$ with $\bar{\Delta}=\left(\begin{array}{cc}\Delta & 0 \\ 0 & \Delta_{c}\end{array}\right)$ having a block diagonal structure, and $\left(\begin{array}{c}\bar{\Delta} \\ I\end{array}\right)$ being a maximal positive subspace.

With a maximal negative subspace of $P_{e}$ of the form $\left(\begin{array}{c}I \\ \Gamma\end{array}\right)$, i.e., for which 
$\left(\begin{array}{c}I \\ \Gamma\end{array}\right)^{T} P_{e}\left(\begin{array}{c}I \\ \Gamma\end{array}\right)<0$ one can apply a "loop shift" of the scheduling block defined by $\widehat{\Delta}=\bar{\Delta}(I-V \bar{\Delta})^{-1}$ according to the transformation

$\left(\begin{array}{l}\widehat{\Delta} \\ I\end{array}\right) \sim\left(\begin{array}{cc}I & 0 \\ -V & I\end{array}\right)\left(\begin{array}{l}\bar{\Delta} \\ I\end{array}\right)=\left(\begin{array}{c}\bar{\Delta} \\ I-V \bar{\Delta}\end{array}\right)$.

The multiplier transforms to

$\tilde{P}_{e}=\left(\begin{array}{cc}I & 0 \\ V & I\end{array}\right)^{T} P_{e}\left(\begin{array}{cc}I & 0 \\ V & I\end{array}\right)=\left(\begin{array}{cc}\tilde{Q}_{e} & \tilde{S}_{e} \\ \tilde{S}_{e}^{T} & R_{e}\end{array}\right)$

with $\tilde{Q}_{e}<0$, as desired.

In order to be able to exploit this fact the "loop shift" should be defined for all $\bar{\Delta}$ and $V$ should be block diagonal with the same pattern as $\bar{\Delta}$, i.e., $V=\left(\begin{array}{ll}V_{p} & 0 \\ 0 & V_{c}\end{array}\right)$. If we can construct such a $V$, then the scheduling variable can be obtained by using the following potential algorithm:

- $\quad$ build the transformed multiplier $\tilde{P}_{e}$ with the transformed variables

$$
\widetilde{\Delta}=\Delta\left(I-V_{p} \Delta\right)^{-1} \text { and } \widetilde{\Delta}_{c}=\Delta_{c}\left(I-V_{c} \Delta_{c}\right)^{-1}
$$

- with this $\tilde{P}_{e}$ construct the shifted scheduling variable $\widetilde{\Delta}_{c}$ according to (12) with $\Delta \rightarrow \widetilde{\Delta}$

- transform back the scheduling variable, i.e., $\Delta_{c}=\widetilde{\Delta}_{c}\left(I+V_{c} \widetilde{\Delta}_{c}\right)^{-1}$

Observe that $\widetilde{\Delta}_{c}$ is an LFT and thus $\Delta_{c}$ is also an LFT using, in general, a repeated block $\left(\begin{array}{cc}\Delta & 0 \\ 0 & \Delta^{T}\end{array}\right)$. To obtain the matrices that define this LFT is a standard computation and it is omitted for brevity.

\subsection{A Maximal Condition}

First, we would like to describe when a block diagonal matrix $V$ exists with the desired properties. Let us suppose that $V_{p}$ is fixed and the question is whether exists a $V_{c}$ such that

$\left(\begin{array}{ll}I & 0 \\ 0 & I \\ V_{p} & 0 \\ 0 & V_{c}\end{array}\right)^{T} P_{e}\left(\begin{array}{ll}I & 0 \\ 0 & I \\ V_{p} & 0 \\ 0 & V_{c}\end{array}\right)<0$.

But this is exactly a problem of type (8), with

$C=\left(\begin{array}{ll}V_{p} & 0 \\ 0 & 0\end{array}\right), \quad A=\left(\begin{array}{l}0 \\ I\end{array}\right), \quad B=\left(\begin{array}{l}I \\ 0\end{array}\right)$

and variable $X=V_{c}$, whose solvability condition is given by the Elimination 
lemma, see the Appendix.

By applying the conditions of the lemma a short computation reveals that the equation is solvable if and only if

$\left(\begin{array}{l}I \\ V_{p}\end{array}\right)^{T} P\left(\begin{array}{l}I \\ V_{p}\end{array}\right)<0$, and $\left(\begin{array}{l}-V_{p}^{*} \\ I\end{array}\right)^{T} \tilde{P}\left(\begin{array}{l}-V_{p}^{*} \\ I\end{array}\right)>0$,

where $P$ and $\widetilde{P}$ are the solution multipliers of the analysis equations.

Remark 1 Observe that applying the same technique for the original problem (13) one can obtain as the solvability condition

$\left(\begin{array}{l}\Delta \\ I\end{array}\right)^{T} P\left(\begin{array}{c}\Delta \\ I\end{array}\right)>0$, and $\left(\begin{array}{c}I \\ -\Delta^{*}\end{array}\right)^{T} \tilde{P}\left(\begin{array}{c}I \\ -\Delta^{*}\end{array}\right)>0$

i.e., exactly the design conditions, as expected.

Actually, we obtain a slightly different result from that from [10]. This analysis ensures the existence of a scheduling variable $\Delta_{c}(\Delta)$ for any extension compatible with $P$ and $\tilde{P}$ and not only for those obtained with a diagonalizing $T$ in (7) as in the original construction. Moreover, $\mathcal{S}_{c}(\Delta)=\left(\begin{array}{c}\Delta_{c}(\Delta) \\ I\end{array}\right)$, can always be assumed without restricting the generality. Since this is a constant Lyapunov function design, continuity of $\Delta_{c}(\Delta)$ does not play any role concerning stability and performance guarantee.

By using a dimension count argument, from the obtained inequalities (15) and the design requirements for $P$ and $\tilde{P}$ it follows that:

Lemma 1 For the loop shifting algorithm it is necessary that there exists a $V_{p}$ satisfying (15) and $\left(\begin{array}{c}\Delta \\ I\end{array}\right)$ and $\left(\begin{array}{c}I \\ -\Delta^{*}\end{array}\right)$ be maximal positive (negative) subspaces of $P$ and $\tilde{P}$, respectively.

Paper [10] gives an algorithm that constructs $\Delta_{c}(\Delta)$ (and not $\mathcal{S}_{c}(\Delta)$ ) if either one of the subspaces is maximal. That algorithm is also hard to implement. However, in order to apply our proposed method, it is necessary that both subspaces be maximal and to exist a $V_{p}$ fulfilling (14).

In what follows, it will be shown that this condition is also sufficient if $V_{p}$ also satisfies (15): then it turns out a loop shift of $\Delta$ is sufficient and a slightly simplified algorithm can be obtained.

The following lemma is a consequence of the Separation Theorems, see the Appendix.

Lemma 2 Let $P \in \mathbb{F}^{(m+n) \times(m+n)}$ be a symmetric or Hermitian matrix with inertia in $(P)=(m, 0, n)$ and let us consider the problem $\left(\begin{array}{l}I \\ \Delta\end{array}\right)^{*} P\left(\begin{array}{l}I \\ \Delta\end{array}\right)<0$.

Then for any fixed solution $\Delta_{0}$ the matrix $I-\Delta_{0}^{*} \Delta$ is nonsingular for all 
solutions $\Delta$.

This lemma ensures that if we find a $\Delta_{0}$ with the properties

$\left(\begin{array}{l}I \\ -\Delta_{0}^{*}\end{array}\right)^{*} P\left(\begin{array}{l}I \\ -\Delta_{0}^{*}\end{array}\right)<0, \quad\left(\begin{array}{l}\Delta_{0} \\ I\end{array}\right)^{*} P\left(\begin{array}{l}\Delta_{0} \\ I\end{array}\right)>0$,

then the choice $V_{p}=-\Delta_{0}^{*}$ will define a loop shift which is well defined for all $\Delta$ of interest.

In what follows it will be shown that under the maximality hypothesis such a $\Delta_{0}$ always exists and it can be computed.

\subsection{Existence of $V_{p}$}

In order to improve readability, some basic facts on maximal negative subspaces and some notations are placed in the Appendix.

Lemma 3 There exists $V_{0} \in \mathbb{F}^{n \times m}$ which satisfies the inequalities

$\left(\begin{array}{l}I \\ V_{0}\end{array}\right)^{*} P\left(\begin{array}{l}I \\ V_{0}\end{array}\right)<0, \quad\left(\begin{array}{l}-V_{0}^{*} \\ I\end{array}\right)^{*} P\left(\begin{array}{l}-V_{0}^{*} \\ I\end{array}\right)>0$.

Proof: The matrix $P$ can be written in the factorized form $P=U^{*} \Lambda J \Lambda U$, where $U$ is a unitary matrix, $\Lambda=\operatorname{diag}\left(\left|\Lambda_{-}\right|^{1 / 2}, \Lambda_{+}^{1 / 2}\right)$ and $J=\operatorname{diag}(-I, I)$.

The second inequality is equivalent to $\left(\begin{array}{l}I \\ V_{0}\end{array}\right)^{*} P^{-1}\left(\begin{array}{l}I \\ V_{0}\end{array}\right)<0$.

Obviously $P^{-1}=U^{*} \Lambda^{-1} J \Lambda^{-1} U$ consequently, $V_{0}$, if exists, has the form

$$
\begin{aligned}
V_{0}=T_{(\Lambda U)^{-1}}\left(L_{1}\right) & =T_{\left(\Lambda^{-1} U\right)^{-1}}\left(L_{2}\right)=T_{U^{*} \Lambda^{-1}}\left(L_{1}\right)=T_{U^{*} \Lambda}\left(L_{2}\right)=T_{U^{*}}\left(T_{\Lambda}{ }^{-1}\left(L_{1}\right)\right) \\
& =T_{U^{*}}\left(T_{\Lambda}\left(L_{2}\right)\right) .
\end{aligned}
$$

This means that $L_{1}=T_{\Lambda^{2}}\left(L_{2}\right)$. Since there exists arbitrary small contraction in the domain of $T_{U^{*}}$, we can choose a contraction $L_{0} \in \operatorname{dom}\left(T_{U^{*}}\right)$, such that $L_{1}=T_{\Lambda}\left(L_{0}\right)$ and $L_{2}=T_{\Lambda}{ }^{-1}\left(L_{0}\right)$ are also contractions. With the choice $V_{0}=T_{U^{*}}\left(L_{0}\right)$ inequalities (16) is satisfied.

The question is, however, whether there exists a matrix $V_{p}$ which satisfies both the inequalities (15) and (14). Unfortunately, this is a more complex question.

As in the proof of Lemma 3 consider the factorization $P=U_{P}^{*} \Lambda_{P} J_{P} \Lambda_{P} U_{P}$ and $\tilde{P}=U_{\tilde{P}}^{*} \Lambda_{\tilde{P}} J_{\tilde{P}} \Lambda_{\tilde{P}} U_{\tilde{P}}$ where $U_{P}$ and $U_{\tilde{P}}$ are unitary matrices. The existence of a $V_{p}$ with the desired properties is guaranteed if there is a contraction $L_{0, p} \in$ $\operatorname{dom}\left(T_{U_{P}^{*}}\right)$ and also a contraction $L_{0, \tilde{p}} \in \operatorname{dom}\left(T_{U_{\widetilde{P}}^{*}}\right)$ such that $V_{p}=T_{U_{P}^{*}}\left(L_{0, p}\right)=$ $T_{U_{\widetilde{P}}^{*}}\left(L_{0, \tilde{p}}\right)$.

This is fulfilled if $L_{0, p}=T_{U_{P}}\left(T_{U_{\widetilde{P}}^{*}}\left(L_{0, \tilde{p}}\right)\right)=T_{U_{P} U_{\widetilde{P}}^{*}}\left(L_{0, \tilde{p}}\right)$. 
Corollary 1 There exists a $V_{p}$ that fulfills both the inequalities (15) and (14) if and only if

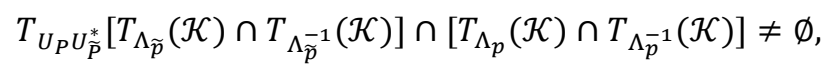

where $\mathcal{K}=\{K \mid K \|<1\}$.

This means that if $T_{U_{P} U_{\widetilde{P}}^{*}}$ maps $\lambda \mathcal{K}$ far from the origin, where $T_{\Lambda_{\tilde{p}}}(\mathcal{K}) \cap$ $T_{\Lambda_{\tilde{p}}}(\mathcal{K}) \subset \lambda \mathcal{K}$ then such a transformation does not exists.

\subsection{Construction Algorithm}

If condition of Corollary 1 is fulfilled there exists a $V_{p}$ that makes possible the transform

$P=\left(\begin{array}{ll}Q & S \\ S^{T} & R\end{array}\right) \mapsto \bar{P}=\left(\begin{array}{ll}\bar{Q} & \bar{S} \\ \bar{S}^{T} & R\end{array}\right)$,

$\tilde{P}=\left(\begin{array}{ll}\tilde{Q} & \tilde{S} \\ \tilde{S}^{T} & R\end{array}\right) \mapsto \hat{P}=\left(\begin{array}{ll}\tilde{Q} & \hat{S} \\ \hat{S}^{T} & \hat{R}\end{array}\right)$

with $\bar{Q}<0$ and $\hat{R}>0$.

With $\bar{P}$ and $\hat{P}$ one can build, using the extension process decribed in (10) and (11), a $\tilde{P}_{e}$ that leads to a solution $\Delta_{c}(\widetilde{\Delta})$ by using (12).

Note that $\Delta_{c}$ can be always put into an LFT form based on the single block $\left(\begin{array}{cc}\Delta & 0 \\ 0 & \Delta^{T}\end{array}\right)$.

Thus we can conclude this paper with summarizing the main result:

Proposition 1 For the loop shifting algorithm to be applicable it is necessary and sufficient that $\left(\begin{array}{c}\Delta \\ I\end{array}\right)$ and $\left(\begin{array}{c}I \\ -\Delta^{*}\end{array}\right)$ be maximal positive (negative) subspaces of $P$ and $\tilde{P}$, respectively, and that there exists a matrix $V_{p}$ which satisfies both the inequalities (15) and (14).

If the conditions of the proposition are fulfilled then the scheduling variables of the controller can be obtained using the following algorithm:

- Compute the transformed matrices

$$
\bar{P}=\left(\begin{array}{ll}
I & 0 \\
V_{p} & I
\end{array}\right)^{T} P\left(\begin{array}{ll}
I & 0 \\
V_{p} & I
\end{array}\right), \quad \hat{P}=\left(\begin{array}{ll}
I & -V_{p}^{*} \\
0 & I
\end{array}\right)^{T} \tilde{P}=\left(\begin{array}{ll}
I & -V_{p}^{*} \\
0 & I
\end{array}\right)
$$

- Build the transformed multiplier $\tilde{P}_{e}$ by the method given in (10) and (11).

- With this $\tilde{P}_{e}$ construct the scheduling variable $\Delta_{c}$ according to the formula (12) with $\Delta \rightarrow \widetilde{\Delta}$, where $\widetilde{\Delta}=\Delta\left(I-V_{p} \Delta\right)^{-1}$. 


\section{Appendix}

For the sake of completeness in this section we summarize the basic results that are used in the separation framework, i.e., in the context of this paper, the LMI approach to the robust control design. These results are formulated in the finite dimensional case, i.e., for matrices. Nevertheless, such assertions can be formulated in a more general operator setting as well.

\subsection{Elimination Lemma}

A fundamental result of the LMI framework in the derivation of the design equations is the Elimination lemma. The conditions of the lemma lead directly to the analysis equations that are the starting point for any controller design.

Lemma 4 Let $Q=Q^{T}$ be a non-singular matrix with inertia in $(Q)=$ $(m, 0, n)$ and let us consider the quadratic matrix inequality

$\left(\begin{array}{c}I \\ C+A X B\end{array}\right)^{T} Q\left(\begin{array}{c}I \\ C+A X B\end{array}\right)<0$.

This inequality has a solution if and only if

$B^{*}\left(\begin{array}{l}I \\ C\end{array}\right)^{T} Q\left(\begin{array}{l}I \\ C\end{array}\right) B<0$

and

$A_{\perp}\left(\begin{array}{c}-C^{T} \\ I\end{array}\right)^{T} Q^{-1}\left(\begin{array}{c}-C^{T} \\ I\end{array}\right) A_{\perp}^{*}>0$

Here $A_{\perp}$ denotes a matrix with $A_{\perp} A=0$ and $A_{\perp} A_{\perp}^{*}>0$ while $B$ denotes an arbitrary basis matrix such that $B B=0$ and that $B^{*} B>0$. For a proof see, e.g., [5], [10].

\subsection{Separation Lemma}

Theorem 2 Let $B \in \mathbb{F}^{n \times m}$ be a fixed matrix and $\boldsymbol{\Delta} \subset \mathbb{F}^{m \times n}$ a compact set of matrices. Then the following are equivalent:

- I-B $\Delta$ is nonsingular for all $\Delta \in \Delta$

- $I-\Delta B$ is nonsingularfor all $\Delta \in \Delta$

- There exists an indefinite matrix $\left.P \in \mathbb{F}^{(n+m) \times(n+m}\right)$

with $\operatorname{in}(P)=(n, 0, m)$ such that

$\left(\begin{array}{l}B \\ I\end{array}\right)^{*} P\left(\begin{array}{l}B \\ I\end{array}\right)>0$ and $\left(\begin{array}{c}I \\ \Delta\end{array}\right)^{*} P\left(\begin{array}{l}I \\ \Delta\end{array}\right)<0, \forall \Delta \in \Delta$.

For a proof of this theorem see, e.g., [7]. 
The duality lemma ensures the simultaneous inequalities

$$
\left(\begin{array}{l}
\Delta \\
I
\end{array}\right)^{*} P\left(\begin{array}{l}
\Delta \\
I
\end{array}\right)>0 \text { and }\left(\begin{array}{c}
I \\
-\Delta^{*}
\end{array}\right)^{*} P^{-1}\left(\begin{array}{c}
I \\
-\Delta^{*}
\end{array}\right)<0
$$

for any $\Delta \in \Delta$, however, at the same time

$\left(\begin{array}{c}I \\ -\Delta^{*}\end{array}\right)^{*} P\left(\begin{array}{c}I \\ -\Delta^{*}\end{array}\right)<0$

does not hold in a general case. If it happens that there is a $\Delta_{0}$ that also fulfills this inequality, the Separation lemma guarantees the invertability of $I+\Delta_{0}^{*} \Delta$ for all $\Delta \in \Delta$.

\subsection{Indefinite Maximal Subspaces}

The following result describes the maximal negative graph subspaces of a symmetric matrix $P$, i.e., all the matrices $Z$ such that

$$
\left(\begin{array}{l}
I_{q} \\
Z
\end{array}\right)^{*} P\left(\begin{array}{l}
I_{q} \\
Z
\end{array}\right)<0
$$

where $P \in \mathbb{F}^{(q+p) \times(q+p)}$ with inertia $\operatorname{in}\left(P_{s}\right)=(q, 0, p)$.

Theorem 3 Let $\mathcal{M}$ be a symmetric matrix such that there is a nonsingular matrix $M$ for which $P=M^{-T} J M^{-1}$, where $J=\operatorname{diag}\left(-I_{m}, I_{n}\right)$. Then all solutions of (21) are given by

$Z=T_{M}(K)$

for $K$ is an arbitrary contraction $(\|K\|<1)$ in $\operatorname{dom}\left(T_{M}\right)$.

For a matrix $M$ partitioned as

$M=\left(\begin{array}{ll}M_{11} & M_{12} \\ M_{21} & M_{22}\end{array}\right)$

the Möbius transformation $T_{M}$ is defined by the equation

$T_{M}(L)=\left(M_{21}+M_{22} L\right)\left(M_{11}+M_{12} L\right)^{-1}$

for $L \in \operatorname{dom}\left(T_{M}\right)=\left\{L:\left(M_{11}+M_{12} L\right)^{-1}\right\}$.

Thus, the parametrization relies on describing $\operatorname{dom}\left(T_{M}\right)$

An exhaustive description of the set $\mathcal{X}_{M_{11}, M_{12}}=\left\{X \mid M_{11}+M_{12} X\right.$ nonsingular $\}$ can be done by using the generalized singular value decomposition (GSVD), however, for our purposes it is sufficient the following result, based on the more familiar singular value decomposition (SVD).

Consider the SVD of $A$ as $A=U_{A} \Sigma_{A} V_{A}^{*}$ with

$U_{A}=\left(\begin{array}{ll}U_{a} & U_{a s}\end{array}\right), \quad \Sigma_{A}=\left(\begin{array}{cc}\Sigma_{a} & 0 \\ 0 & 0_{a s}\end{array}\right), \quad V_{A}=\left(\begin{array}{ll}V_{a} & V_{a s}\end{array}\right)$. 
and that of $B=U_{B} \Sigma_{B} V_{B}^{*}$ with

$U_{B}=\left(\begin{array}{ll}U_{b} & U_{b s}\end{array}\right), \Sigma_{B}=\left(\begin{array}{ll}\Sigma_{b} & 0 \\ 0 & 0_{b s}\end{array}\right), V_{B}=\left(\begin{array}{ll}V_{a} & V_{b s}\end{array}\right)$.

With these notations one has:

Lemma 5 The matrices

$X_{0}(\gamma)=V_{B}\left(\begin{array}{cc}0 & \gamma \Sigma_{b} U_{b}^{*} U_{a s} \\ 0 & 0\end{array}\right) V_{A}^{*}$.

make $A+B X_{0}(\gamma)$ nonsingular for every $\gamma \neq 0$.

Moreover, for $\gamma^{*} \leq \frac{1}{\|B\|}$ the matrix $X_{0}(\gamma)$ is contraction for all $|\gamma|<\gamma^{*}$.

More details on the construction and the proofs can be found in [14]. A general overview on indefinite matrix analysis can be found in [4].

\section{Conclusions}

This paper investigates the possibility of constructing the scheduling block of a qLPV controller explicitly, i.e., in form of an LFT. It was shown that if both the primary and dual equations lead to maximal indefinite subspaces and a coupling condition holds, then the problem can be solved. In addition, a constructive algorithm was provided to build the needed scheduling variables. Currently, an efficient test of the coupling condition is under investigation.

The efficient construction of the scheduling block, in a general case, i.e., under the nonrestrictive inertia conditions of the full-block S-procedure, is still an open problem and it is the subject to future research.

\section{Acknowledgement}

The research has been conducted as part of the project TÁMOP-4.2.2.A-11/1/KONV-2012-0012: Basic research for the development of hybrid and electric vehicles. The Project is supported by the Hungarian Government and co-financed by the European Social Fund.

\section{References}

[1] A. Packard and G. Balas. Theory and Application of Linear Parameter Varying Control Techniques. American Control Conference, Workshop I, Albuquerque, New Mexico, 1997

[2] P. Gahinet and P. Apkarian. An LMI-based Parametrization of All $H_{\infty}$ Controllers with Applications. Proceedings of the $32^{\text {nd }}$ IEEE Conference on Decision and Control, pp. 656-661, 1993

[3] P. Gahinet, P. Apkarian, and M. Chilali. Affine Parameter Dependent Lyapunov Functions and Real Parameter Uncertainty. IEEE Transactions on Automatic Control, Vol. 41, pp. 436-442, 1996 
[4] I. Gohberg, P. Lancaster and L. Rodman. Indefinite Linear Algebra and Applications. Birkhauser, 2005

[5] A. Helmersson. IQC Synthesis Based on Inertia Constraints. Proceedings of the $14^{\text {th }}$ IFAC World Congress, Bejing, China, pp. 163-168, 1998

[6] T. Iwasaki and R. E. Skelton. All Controllers for the General $H_{\infty}$ Control Problem: LMI Existence Conditions and State Space Formulas. Automatica, 300 (8):0 1307-1317, 1994

[7] T. Iwasaki. Recent Advances on LMI Methods in Control, chapter Control Synthesis for Well-Posedness of Feedback Systems, pp. 229-247, SIAM, 2000

[8] T. Iwasaki and G. Shibata. LPV System Analysis via Quadratic Separator for Uncertain Implicit Systems. IEEE Transactions on Automatic Control, Vol. 46, pp. 1195-1208, 2001

[9] C. W. Scherer. Recent Advances on LMI Methods in Control, chapter Robust Mixed Control and LPV Control with Full Block Scalings, pp. 187-208, SIAM, 2000

[10] C. W. Scherer. LPV Control and Full Block Multipliers. Automatica, 270 (3):0 325-485, 2001

[11] C. W. Scherer and S. Weiland. Linear Matrix Inequalities in Control, Dutch Institute of Systems and Control (DISC) 2005

[12] J. Veenman and C. W. Scherer. On Robust LPV Controller Synthesis: A Dynamic Integral Quadratic Constraint based Approach, 49 ${ }^{\text {th }}$ IEEE Conference on Decision and Control, Atlanta, GA, USA, 2010

[13] Z. Szabó, P. Gáspár and J. Bokor, A Novel Control-oriented Multi-Affine qLPV Modeling Framework, Proc. of the $18^{\text {th }}$ Mediterranean Conference on Control and Automation, Marrakech, Maroc, pp. 1019-1024, 2010

[14] Z. Szabó, Zs. Biró and J. Bokor. All Controllers for an LPV Robust Control Problem,' Proceedings of the $7^{\text {th }}$ IFAC Symposium on Robust Control Design, Aalborg, Denmark, 2012

[15] F. Wu, X. H. Yang, A. Packard, and G. Becker, Induced $\mathbb{L}^{2}$ Norm Control for LPV Systems with Bounded Parameter Variation Rates. International Journal of Nonlinear and Robust Control, Vol. 6, pp. 983-998, 1996

[16] F. Wu. A Generalized LPV System Analysis and Control Synthesis Framework. International Journal of Control, 740 (7):0 745-759, 2001

[17] F. Wu and B. Lu. On Convexified Robust Control Synthesis. Automatica, 400 (6):0 1003-1010, 2004

[18] F. Wu and K. Dong. Gain Scheduling Control of LFT Systems Using Parameter Dependent Lyapunov Functions. Automatica, Vol. 42, pp. 39-50, 2006 